\title{
The Feasibility Study of the School-Based Curriculum of Wushu Rank System on Junior High School
}

\author{
Sheng Chen ${ }^{1, a, *}$, Haidan $\mathrm{Li}^{2}$ and Rui $\mathrm{Xiao}^{3}$ \\ ${ }^{1}$ College of Physical Education, Chengdu University, Chengdu, Sichuan, China \\ ${ }^{2}$ Qianhai School in Nanshan District, Shenzhen, Shenzhen, Guangdong, China \\ ${ }^{3}$ School of Literature and Journalism, Sichuan University, Chengdu, Sichuan, China \\ a787345913@qq.com \\ *corresponding author
}

Keywords: Wushu Rank System, School-Based Curriculum, Curriculum Development

\begin{abstract}
In this paper, using the literature and logic analysis method, combining the concep t with Wushu Rank System, school-based curriculum, school-based curriculum of Wushu to d efinition the school-based curriculum of the Wushu Rank System: based in the school, throu gh the secondary development of Wushu course, choose the contents of the Wushu Rank Sy stem as school-based curriculum of Wushu Rank System teaching content, through the knowl edge fusion of symbiosis, the implementation of curriculum development and curriculum deci sion-making course.The need for the development of excellent traditional cultural heritage an $\mathrm{d}$ the "three-level curriculum management system" provide the guarantee for the development of the school-based curriculum of Wushu Rank System.Finally, three strategies are put forw ard: To support the teaching by improving the teaching ability of the section system of teac hers,enrich the campus activities of the Wushu Rank System and establish the system guaran tee of the school Wushu Rank System.
\end{abstract}

\section{Concepts}

\subsection{Wushu Duanwei System}

The Duanwei System of Wushu (Chinese martial arts) can be traced back to Wushu Talent Selection System in the Tang Dynasty: to select talents mainly by their levels of martial arts. With the progress of the times, the Chinese Wushu Association set up Wushu Duanwei System in 1998 with the aim of promoting the development of wushu and establishing a standardized wushu practice system and wushu technical evaluation system. The evaluation targets of the Wushu Duanwei System embrace the number of years practicing wushu and conducting martial arts activities, the mastery level of wushu skills and theories, research achievements, wushu ethics, and contribution to the development of wushu. The whole system consists of three Dangs, nine Duans and Twelve levels[1]. The Wushu Duanwei is formulated and implemented on the basis of the overseas experience on promoting Go and taekwondo in order to link the wushu evaluation system to the world. This system is an important carrier for standardizing wushu in the big picture of "Big Wushu". In May 2014, the General Administration of Sport of China promulgated the 10-year Plan for the Promotion of Wushu Duanwei System, which puts Wushu Duanwei System entering campus as a priority for promotion [2]. This plan represents an in-depth reform and top-level design to create the general direction in the public sports service system, to popularize Wushu Duanwei System, and to 
increase the grassroots base. To implement Wushu Duanwei System in campuses can not only allow students to understand and study wushu, thus strengthening their awareness of national culture, but also achieve a win-win situation of promoting both wushu modernization and students' physical and mental growth. If the learning environment is put in good use and the cohesive spirit of national culture is vigorously publicized, schools will surely become a successful source for wushu promotion. Therefore, to promote and implement Wushu Duanwei System in schools is an inevitable trend in today's society, and also a long-term process.

\subsection{School-based curriculum}

The word "curriculum" began as a Latin word "currere", which means the course of a race. A curriculum consists of education objectives, learning contents and studying activities. As one part of schooling process, a curriculum is an educational program of multi-level organizational structures, which contains schooling plans and information to guide schooling and teaching activities [3]. The school-based curriculum takes teachers as its main body in line with the school's guidelines. It's an educational program designed for achieving the education objectives in the process of implementing the national and local curriculum by assessing the local students' needs in a scientific way and by making full use of the curriculum resources at local communities and schools. Explicit school-based curricula refer to those that are produced, implemented and evaluated by all, some or individual teachers, while implicit school-based curricula refer to teachers' tendency of extending curriculum in the process of implementing the national curriculum [4]. According to the national curriculum plan, schools are in charge of 5\% of total class hours for their own course development. These self-developed courses are called school-based curricula and it is a continuous and dynamic process to improve the curriculum [5]. The broad sense of school-based curriculum development includes the curriculum that is adapted for the school on the basis of the national and local curriculum. It aims to fit the actual situation of the school and to meet the needs of students. In addition, the broad sense also means the school-based adaptation of the national curriculum plan according to its actual situation. Teachers can still make their own decisions on curriculum topics such as curriculum resources, unit progress, teaching sequence, teaching methods, and evaluation methods according to the characteristics and conditions of the school, which includes both the development of school-based curriculum and the school-based implementation of the national and local curriculum.

\subsection{Wushu School-based Curriculum}

Physical education curriculum is mainly a technical practical curriculum. The development of school-based physical curriculum is essentially the school's "doing its own thing" - to design personalized curriculum within the framework of the national Sports and Health Curriculum Standards. It should include two main points. One is to personalize and implement the school-based national and local curriculum. It is a process for sports teachers to improve and recreate national and local curriculum through selection, adaptation, integration, supplement and expansion in order to be more consistent with the characteristics and needs of students, schools, and society. The other is new curriculum designed and developed by schools. It is a process for schools and sports teachers to take local curriculum resources into consideration and to create courses that fit students when implementing national and local curriculum. At present, many schools have optional sports courses and activities based on their own characteristics and students' needs. These courses and activities are budding or elementary forms of school-based curriculum. However, because schools or sports 
teachers are lack of knowledge on curriculum theory and school-based curriculum, they are unaware of curriculum development. Wushu school-based curriculum is developed and implemented in schools with Chinese martial arts as its main content. Its development calls for principals, teachers, curriculum experts, students, and parents to work together on curriculum planning, implementation and evaluation [6].

\subsection{School-based Curriculum of Wushu Duanwei System}

Wushu Duanwei System solves the problem of how and what to teach about martial arts in Junior High and secondary schools. The System is logically and systematically designed as there are different teaching contents and corresponding level systems for students at all ages. It demonstrates the scope, depth, difficulty and validity of teaching content and promotes the combination of wushu teaching and evaluation. The straightforward Wushu Duanwei System can help build wushu school-based curriculum in schools by offering institutional references to wushu teaching. Wushu, as part of traditional Chinese culture, is very popular among students. However, for many years, schools teach wushu in the mode of "broadcast exercises - basic skills - routine", which usually don't take students' needs into account. Students' needs, expectations and experience on wushu are often detached from the edge of wushu teaching. Such wushu curriculum centering on textbooks or students can often generate students' love for wushu, but not for wushu class. Meanwhile, the limited expertise of sports teachers makes it hard to realize the teaching objectives required in the new curriculum and even harder to promote wushu. Therefore, we need a path to the growth peak and the development of wushu school-based curriculum can be the starting point. In the curriculum, Wushu Duanwei System is multilevel and systematic. Wushu teaching combines combat and practice and uses the preserved 5\% time and space. After the secondary development of wushu curriculum, schools choose part of Wushu Duanwei System together with other subjects as teaching material. It is an easily operating strategy to first implement on a small scale, and then use the development and implementation of school-based curriculum to combine points and planes. As a result, teachers enhance their proficiency, students express their personalities and schools highlight their unique strengths.

\section{Feasibility analysis on the establishment of the school-based curriculum of Wushu Duanwei System in Junior High schools}

\subsection{Carry forward outstanding traditional culture in schools}

On January 25, 2017, the General Office of the CPC Central Committee and the General Office of the State Council issued a notice On Implementing the Program of Inheriting and Developing Outstanding Traditional Chinese Culture. As the economy and society have been undergoing profound changes, various ideological and cultural exchanges, interactions and conflicts happen frequently. Therefore, there is an urgent need for Chinese people to deepen their understanding of the importance of outstanding traditional Chinese culture, and further enhance cultural awareness and self-confidence. The Notice centers on the fundamental task of cultivating students' high moral values and follows students' cognitive and learning process. It fully integrates traditional culture into ideological and moral education, cultural and intellectual education, arts and physical education as well as social practice education. It promotes drama, calligraphy, elegant art, and traditional sports to enter into the campus. The campus will become a hot spot for inheriting traditional Chinese culture, and the value of Junior High and secondary students will represent the future of the country as it says 
"the young is strong and the country strong". Wushu is the national quintessence with rich cultural forms. To promote wushu in Junior High schools, the starting point for basic education, can not only allow students to build strong and healthy body, but also absorb the nutrition of traditional culture so as to shape their personalities and increase their national self-confidence. Gao Xiaojun, director of the Wushu Sports Management Center of the General Administration of Sport, said: "The Wushu Duanwei System is an important method and carrier for Wushu inheritance, promotion and popularization. It acts as a nervous system for the development of Chinese martial arts and plays a crucial role in wushu's future, healthy development and global promotion."

\section{2 "Three-level curriculum management system" provides guarantee for the school-based curriculum development of Wushu Duanwei System in Junior High schools}

The Guide to School Curriculum Management clearly states that schools should "study students' needs for diversified development, develop or select courses suitable for the characteristics of the school and available for students to choose", and puts forward clear requirements for how to develop and implement school-based curriculum. This ensures the development of school-based sports curriculum. At present, a series of aerobics featuring wushu for Junior High and secondary school students produces a good result. After practicing the wushu aerobics, students show their strong interest in wushu. The development and implementation of school-based curriculum, which should be feasible and practical, requires human and material resources. The textbook of the school-based curriculum for Wushu Duanwei System is more in line with students' interest and physical and mental conditions; the assessment and evaluation mechanism is encouraging. As the teaching content of Duanwei System is the same as what is promoted in society, wushu practitioners of high levels are also qualified to teach in schools. Therefore, schools can employ those practitioners. This is a way of integrating all resources from society, communities and schools to solve the issue of faculty shortage. The development and implementation of the school-based curriculum of Wushu Duanwei System is a bottom-up process following related paper instructions from the General Administration of Sport of China and the Department of Education.

\section{Development Strategies for the school-based curriculum of Wushu Duanwei System in Junior High schools}

\subsection{Enhance teacher's teaching capacity to support teaching}

Faculty is a powerful guarantee for the effective implementation of Wushu Duanwei System. To solve the issue of faculty shortage in China, the Wushu Sports Management Department can work with the Education Department to formulate continuing education trainings on the System according to the teacher resources of all places to enhance the teaching capacity. Schools can employ wushu practitioners of high levels from society and also carry out the project of "letting higher education and social forces join sports and art education in Junior High schools" to provide platforms for the System development in order to share and connect resources. The strategy is to let institutions of higher learning and research institutes participate in the process of initiating, maintaining, and further developing the curriculum. The importance of the strategy is also proved by the successful story at Xishan High School. However, using only external forces to develop school-based sports curriculum is not a long-term solution. Instead, the schools must enhance the internal ability of developing curriculum, especially the professional level of sports teachers, so as to increase the self-hematopoietic function and form a virtuous circle. 


\subsection{Enrich Wushu Duanwei System activities in the campus}

Wushu aerobics are one of the important parts of sports education in Junior High and secondary schools. Wushu aerobics enable students to have an intuitive understanding of wushu. Schools with qualified conditions can implement the wushu school-based curriculum and Duanwei System is the answer to what to teach in schools. Besides, schools can offer Duanwei System study, organize practitioners to perform, publicize evaluation of the System and play wushu documentaries after class. By doing so, schools gain their unique feature based on Wushu Duanwei System, and use the benefits of growth pole to create good wushu atmosphere in campuses from points to planes and to more dimensions [7]. When we sow a seed of "reform" in the soil, this seed will germinate and grow. If we cultivate proper soil around it, this seed will spread like growing grassroots. Therefore, we should take the development of wushu school-based curriculum as a breakthrough point, sow the seeds of this reform, let it spread and produce a grassroots effect so as to achieve an all-round Wushu reform in schools.

\subsection{Establish the organizational guarantee for Wushu Duanwei System in schools}

Junior High and secondary schools teach Duanwei System through school-based wushu curriculum. The teaching process requires the horizontal coordination among curriculum research communities. This can ensure that the school-based curriculum is developed in a scientific way and implemented efficiently. This horizontal coordination includes the work of teaching, guaranteeing equipment needed, organizing cultural and artistic performances by practitioners, and publicizing the Duanwei System from multiple aspects. The evaluation of the Duanwei System needs to be combined with effective management of Duanwei System working committees, offices, and test centers. This management, on the basis of horizontal coordination, will ensure that the test center play its role and the evaluation can be conducted efficiently.

\section{Summary}

Since the introduction of Wushu Duanwei System in 1998, the publicity and promotion of the System has been in the situation where much is said but little is done. This is due to the lack of good interaction between the Ministry of Education and the Wushu Sports Management Center of the General Administration of Sport. Other causes are related to the local wushu basics, the decision-making awareness of the leaders, and professional quality of relevant staff. The combination of the Wushu Duanwei System and school-based curriculum is based on the current wushu promotion in Junior High schools, the faculty situation for Duanwei, and the characteristics of the System itself. In Junior High schools, we carry out national culture education to inherit culture. As wushu is an outstanding traditional Chinese culture, it'll be a focus for teachers to work out how to prosper wushu culture through sharing wushu and other resources, and how to maximize the role that wushu can play in cultural education. To combine Wushu Duanwei System and school-based curriculum is feasible and practical both theoretically and operationally. To set up Duanwei teaching objectives and evaluation that meet physical and mental needs of students at all ages will help achieve the sustainable development of wushu.

\section{References}

[1] Wushu Sports Management Center of the General Administration of Sport of China, Chinese Wushu Association, Wushu Research Institute of the General Administration of Sport of China. 
Chinese Martial Arts Rank Manual, Revised Edition [Z]. 2013, 12.

[2] Sports General Administration of Sport of China, All-China Sports Federation. 10-year Plan for the Promotion of Wushu Duanwei System (2014-2023) [Z]. 2015: 176.

[3] Dong Cuixiang. Research on the Theory and Practice of School-based Sports Curriculum Development in Junior High and Secondary schools in China [D]. Beijing Sport University. 2004:20. [4] Wang Binhua. School-based Curriculum Theory [M]. Shanghai: Shanghai Education Press. 2000, 12.

[5] Xu Yuzhen. School-based Curriculum Development: Conceptual Interpretation [J]. Curriculum, Teaching Materials, Teaching Methods. 2001 (4): 12-18.

[6] Huang Yuquan. Curriculum and Teaching Theory [M]. Beijing: Higher Education Press. 2002,03. [7] Cai Shaohong. The Construction of the Green Growth Pole in the Western Ecologically Fragile Region-A Research Based on the Circular Industrial Cluster Model [M]. Beijing: People's Publishing House, 2015,03. 